Article

\title{
A Spatial Analysis Approach for Evaluating the Service Capability of Urban Greenways-A Case Study in Wuhan
}

\author{
Junwen Bai ${ }^{1,2}$, Penglin Zhang ${ }^{1,2, *} \mathbb{D}$, Juan Du ${ }^{1, *}$ and Zheng $\mathrm{Li}^{1}$ \\ 1 School of Remote Sensing and Information Engineering, Wuhan University, Wuhan 430079, China; \\ JorvenBai@whu.edu.cn (J.B.); mzzw@whu.edu.cn (Z.L.) \\ 2 Collaborative Innovation Center of Geospatial Information Technology, Wuhan University, \\ Wuhan 430079, China \\ * Corresponding author: zpl@whu.edu.cn (P.Z.); dujuan_rs@whu.edu.cn (J.D.); \\ Tel.: +86-27-68777175 (P.Z.); +86-186-0272-0952 (J.D.)
}

Academic Editors: Ozgun Akcay, Carlos Granell Canut and Wolfgang Kainz Received: 29 March 2017; Accepted: 5 July 2017; Published: 8 July 2017

\begin{abstract}
A greenway is a low-speed road system built on high-level afforestation, and this system serves as a venue for sightseeing, relaxation, and exercise. In China, greenway planning and construction have been actively and successfully implemented to strengthen the construction of urban human settlements. However, a specific method has yet to be used to assess the service capability of urban greenways. On the basis of geographic information systems and geographical spatial data, we propose an approach to evaluate the overall service capability of urban greenways based on three different aspects. In the first aspect, a buffer-based service-level analysis method is applied to statistically analyze population and residential quarter areas within a greenway service coverage area. This aspect can also indicate the service level and scope of urban greenways. In the second aspect, minimum distance-based accessibility analysis method is utilized for the graduation statistics of residential quarter areas in different ranges of reach distance. This aspect can further reveal the service convenience of urban greenways. In the third aspect, a calculation model is built on the basis of regular grids, to analyze the value of comprehensive service capability in each grid, and to produce a spatial distribution map of the comprehensive service capability of urban greenways. This aspect can describe the service quality of urban greenways. The analysis results of these aspects can be integrated to identify service conditions in which an urban greenway is available to urban populations and residential zones, and to obtain the comprehensive service capability value of greenways. This approach can also emphasize the limitations of greenway construction, and thus help urban planners and decision-makers create optimized urban greenway designs.
\end{abstract}

Keywords: greenway; service capability; accessibility; regular grid; spatial distribution

\section{Introduction}

A greenway is an important element of a green open space [1] and relevant space carrier that can satisfy the requirements of constructing urban and residential ecological environments. A well-planned urban greenway system provides a new low-carbon lifestyle that allows individuals to interact closely with nature $[2,3]$. 
An urban greenway offers various services, including leisure and recreation, ecological and economic development, culture and education, and esthetics [4]. These different aspects provide the public with a place to run, ride and travel, clean up air and water in cities, reduce landscape fragmentation, and improve urban environments. Urban greenway also helps increase tourism income, enhance surrounding real estate value, and reduce disease incidence and medical expenses. In addition, urban greenway is characterized by a remarkable educational and esthetic value, because it can connect historical and cultural relics with natural scenic areas to ensure that tourists can appreciate natural, historical, and cultural landscapes.

According to the concept of public service facility, service capability refers to the practical effects generated after certain facilities provide services to the public [5]. Urban greenway belongs to the category of urban public service facilities. Thus, greenway service capability refers to the supply capability of greenways in space.

Since pioneer studies on modern greenways in China were performed in the 1990s [6], many studies on greenway theory and practice cases have been reported [7]. Some studies have mainly focused on greenway planning and construction, and suitability and feasibility analyses have been conducted using ecological, investigative, and geographic information system (GIS) overlay analysis methods [8-11]. Other studies have examined the effects and functions of a greenway network by using a series of statistical approaches based on post-occupancy evaluations, importance-performance analysis models, and questionnaire surveys [12-15]. However, previous studies on greenways failed to assess the service capability of urban greenways. This initiative should be performed because the limitations of greenway construction can be identified, and urban planners and decision-makers can be provided with some scientific recommendations for optimum greenway construction. In our study, an approach based on spatial analysis and statistical techniques was proposed to evaluate the service capability of a greenway network quantitatively and objectively. A buffer-based analysis method was applied to divide an urban greenway service region into different service level areas. Population statistics and residential quarter area statistics were then conducted on the basis of the division result of the greenway service region. A minimum distance-based analysis method was also utilized to group the residential quarters into various accessibility levels. Residential quarter area statistics was also performed in each of the accessibility levels. A comprehensive service capability calculation model for urban greenways was built by considering all relevant factors. Our results demonstrated that the proposed model, which is based on regular grids with a size of $1 \mathrm{~km} \times 1 \mathrm{~km}$, can be used to calculate the comprehensive service capability of an urban area greenway, and can provide new solutions and concepts for the quantitative evaluation of the service capability of other urban infrastructures.

\section{Study Area and Dataset}

The selected study area is Wuhan, which is located in the eastern part of Hubei Province, and is a major city in central China. Its geographical position is $113^{\circ} 41^{\prime}-115^{\circ} 05^{\prime} \mathrm{E}$ and $29^{\circ} 58^{\prime}-31^{\circ} 22^{\prime} \mathrm{N}$, and its area is $8494 \mathrm{~km}^{2}$. According to the Sixth National Census Data, its resident population is 9.79 million.

Urban greenways in Wuhan are mainly used for recreation, leisure, exercise, and sightseeing. These recreational greenways have been built on the basis of existing natural elements, such as natural mountain paths, shores, and greenbelts. Some parts of these recreational greenways are connected to parks, scenic spots, and historical sites. As such, some of them also link historical humanity and cultural landscapes. Recreational greenways also dominate the urban greenway system in Wuhan in accordance with local conditions. In this urban greenway system, other urban greenways can also form a connection between historical and cultural sites, although some of them belong to natural corridors. Figure 1 shows some of the sceneries in Wuhan greenway. 

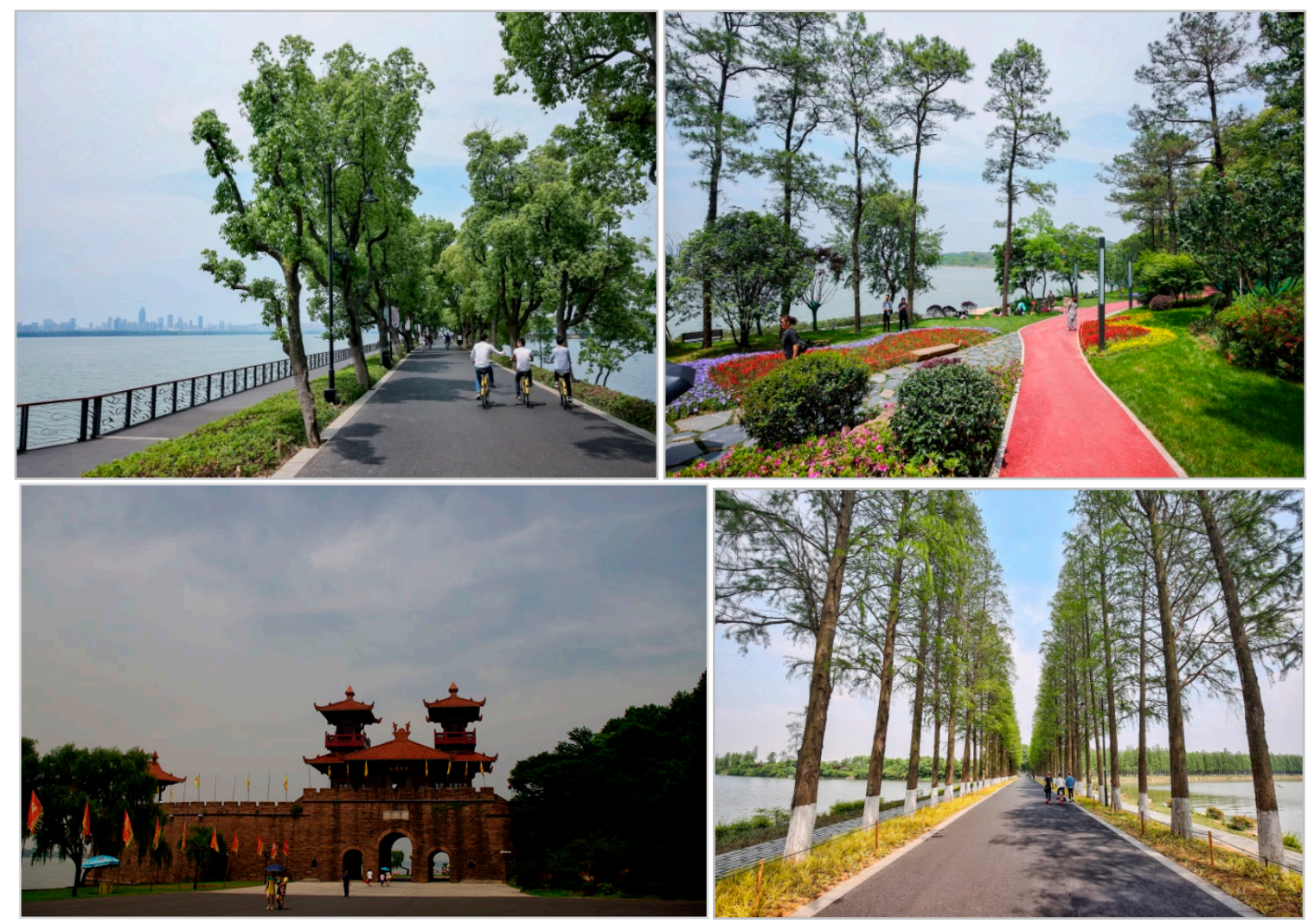

Figure 1. Some sceneries in Wuhan greenway (Source: The left down one from http://blog.sina. com.cn/s/blog_51e387ef0100thc1.html. Others from https://m.weibo.cn/status/4103092829771911; accessed on 9 May 2017).

The experimental datasets used in this study included data from Wuhan greenway plans, national geographic state surveys, Sixth National Census, public transport points, and service facility points. The national geographic state survey data of Wuhan City were mainly used to provide residential quarter information, which was referred to as residential quarter data in this paper, and combined the service facility point information. The data from the Sixth National Census of Wuhan City were utilized to determine the population density information, which was designated as population density data in this paper. The public transport point data included information from passengers, buses, and subway stations. The service facility point data consisted of public service and government service facility points. The public service facility point data of Wuhan comprised parks, green squares, A-grade tourist districts, museums, libraries, places of interest, nursing homes, and other similar locations. The government service facility point data constituted police units, fire agencies, and community points. The urban greenway system can link urban public green systems and tourism resources. In this context, transportation convenience and some government safety facilities are necessary to provide enhanced services.

In 2012, the Land Resources and Urban Planning Bureau of Wuhan developed a greenway system intended to create a green and habitable Wuhan, and to improve the quality of the city and the living quality of the citizens. This plan presented the layout of the Wuhan greenway network (Figure 2). The total length of greenways in Wuhan is approximately $2200 \mathrm{~km}$. Of the total length of greenways, about $450 \mathrm{~km}$ is allocated for the full length of greenways in the main urban areas, and nearly $1750 \mathrm{~km}$ is part of the greenways in suburban areas. The greenway system planning in Wuhan also involved the development goals of Wuhan greenway construction. These development goals suggested that Wuhan greenways can be respectively reached in 5-10, 15-20, and 30-45 $\mathrm{min}$ in the community, main urban areas, and suburban areas by the end of the 12th Five-year Plan (2015). 


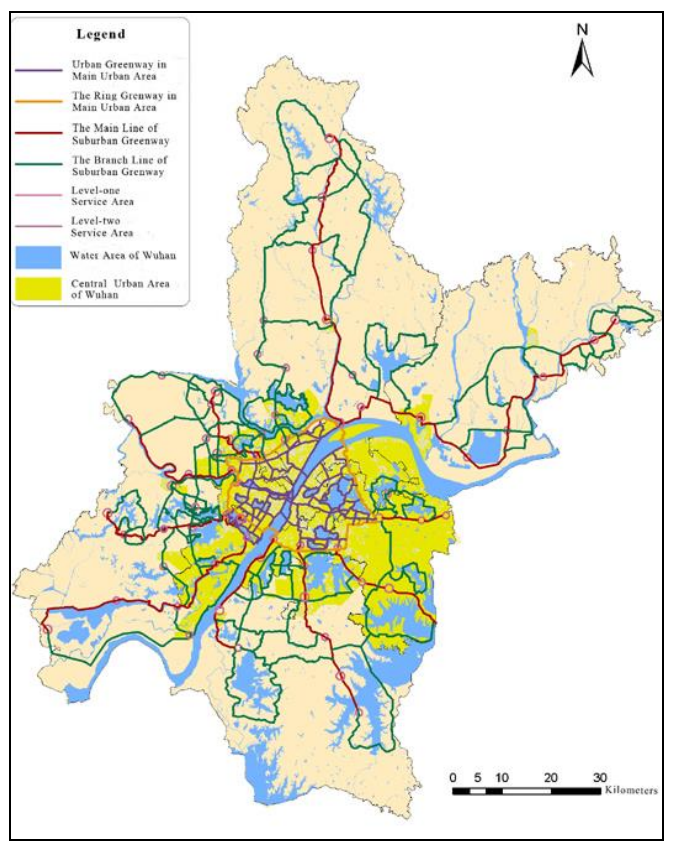

Figure 2. Layout map of Wuhan greenway network.

\section{Methodology}

\subsection{Overview of the Method}

The complete evaluation process of urban greenway service capability is shown in Figure 3. First, two preparation steps, namely, experimental data processing and greenway classification, must be performed. Afterward, the service capability of urban greenway can be evaluated by considering the three aspects. In the first aspect, a buffer-based service level analysis method is used for the graduation statistics of the population and residential quarter areas within the service coverage area of the greenway. In the second aspect, the minimum distance-based accessibility analysis method is utilized for the graduation statistics of residential quarter areas in different ranges of reach distance. In the third aspect, a calculation model is built on the basis of the regular grids and various factors to examine the comprehensive service capability of urban greenways.

Two key points should be explained about the process shown in Figure 3:

(1) In the experiment data processing stage, all the data involved in the operation were unified in the same coordinate system and space scale using technologies, including coordinate transformation and data registration.

(2) The main object of greenway classification is to obtain greenway classes with distinct locations. Moreover, the greenway classes obtained will be used in buffer-based service level analysis. The greenway system was divided into three classes according to the greenway system planning in Wuhan. These classes are communities, main urban areas, and suburban areas. Community greenways and main urban greenways are located in the central urban area; hence, no significant differences exist between the two types of greenway. This paper chooses a similar classification method to divide the greenway system into three classes. The ring greenway in the main urban area was used as a dividing line. The greenways within the ring were set to Level 1; the ring greenway itself was set to Level 2; and the greenways outside the ring were set to Level 3 . The result of greenway classification is shown in Figure 4. 


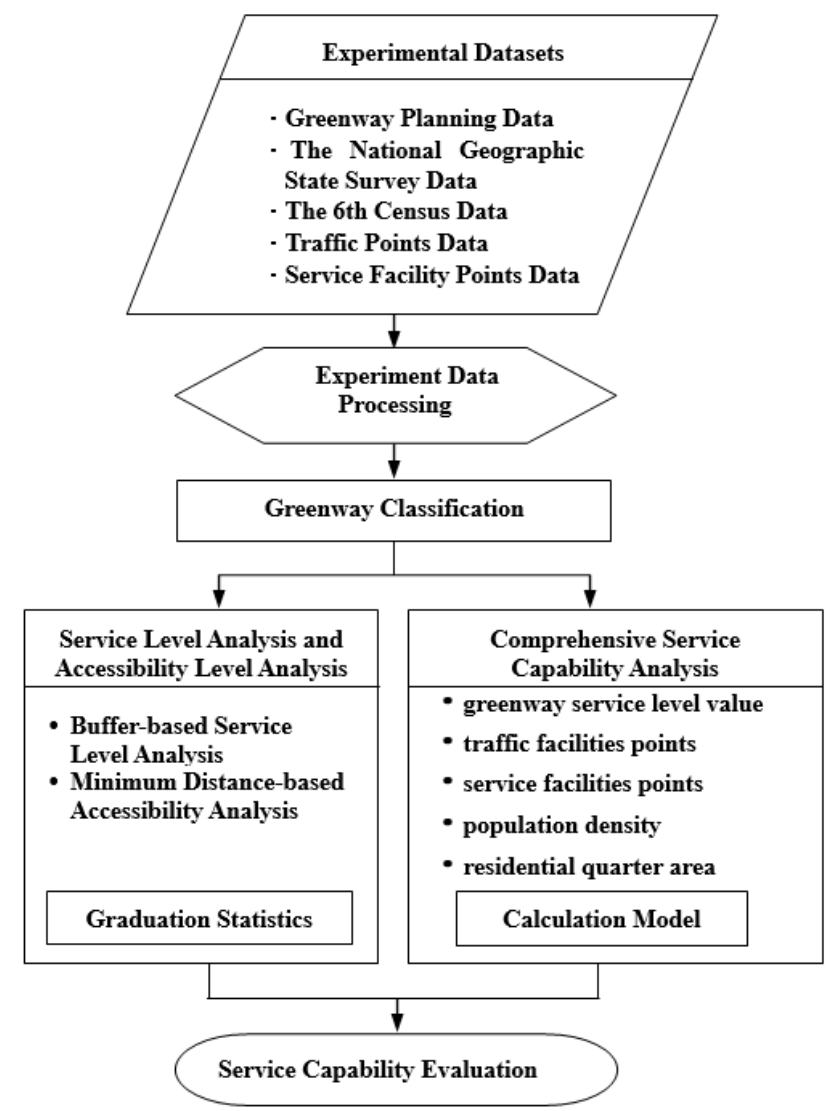

Figure 3. Flow chart of the service capability evaluation of the urban greenway system.

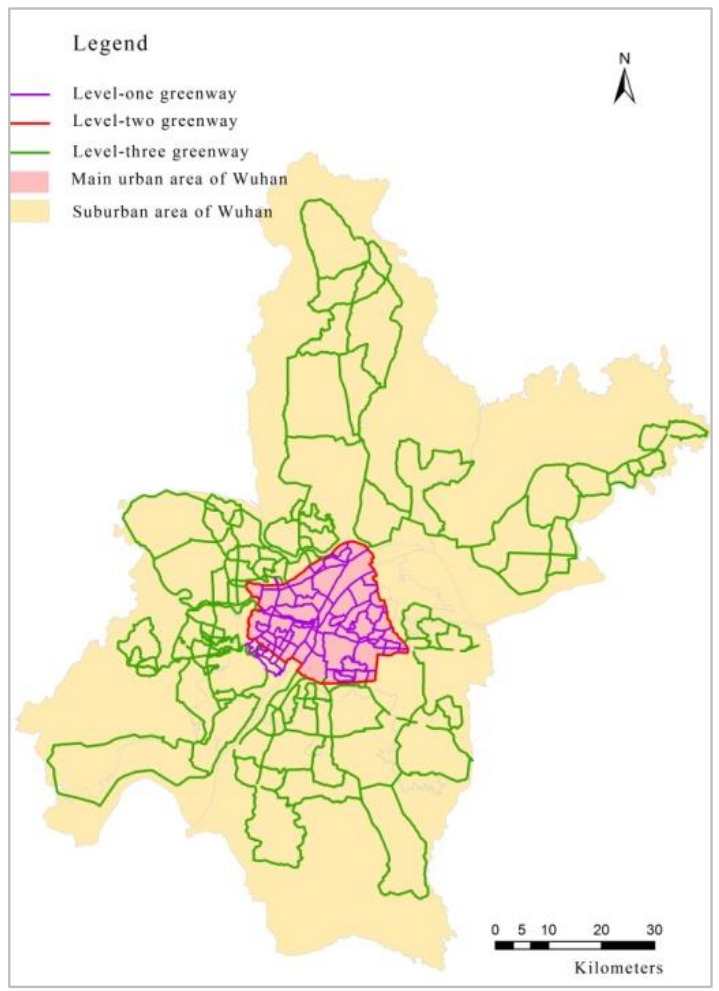

Figure 4. Layout map of the three classes of Wuhan greenways. 


\subsection{Buffer-Based Service Level Analysis}

The scope of services is an important aspect of assessing service capabilities, which reflects the range of greenway services. Therefore, to evaluate the service capacity of urban greenways, it is necessary to analyze its scope of services. The buffer-based service level analysis method can effectively determine the regional scope, population range and residential area of linear feature services. Thus, this article uses this method from the perspective of the scope of service to evaluate the urban greenway service capacity.

According to the time-cost planned in the greenway system planning in Wuhan stated in Section 2, the time of arrival can be assigned for each class of greenway. Level 1 greenways are located in the main urban area, and mostly consist of community greenways. Therefore, the arrival time range of Level 1 greenways was set to $5-10 \mathrm{~min}$. The Level 2 greenway is the ring greenway in the main urban area, which belongs to main urban greenways. Thus, the arrival time range of the Level 2 greenway was set to 15-20 min. Level 3 greenways are located outside the main urban area and consist of suburban greenways. Therefore, the arrival time range of Level 3 greenways was set to $30-45 \mathrm{~min}$.

After the arrival time range of the three classes of greenway is determined, the greenway service radius can be calculated when the walking speed is provided. Thus, the service radius of each greenway class is computed by assuming that the average walking speed is $1 \mathrm{~m} / \mathrm{s}$ [Equation (1)]. Apparently, the service radii of Levels 1, 2, and 3 greenways are 600, 1200, and $2700 \mathrm{~m}$, respectively. The service radius is determined as follows:

$$
R_{i}=\bar{v} \cdot t_{\text {imax }}
$$

where $R_{i}$ is the service radius of Level $i$ greenway, $\bar{v}$ is the human average walking speed, $t_{\text {imax }}$ is the upper limit of arrival time of Level $i$ greenway, and $i$ is 1,2 , or 3 .

Subsequently, the relative service level value for each class of greenway should be set. The relative service level value can be used to represent the greenway service level. However, the value does not represent the accurate value of greenway service level. This value is simply a reference, which indicates that a high relative service level value corresponds to the service that can be provided by this kind of greenway. Figure 4 illustrates that Level 1 greenway is located in the main urban area and characterized by an apparent geographical advantage. Thus, improved services can be theoretically provided by Level 1 greenway compared to others. Similarly, the services that can be obtained from Level 2 greenway are better than those from Level 3 greenway. Thus, the relative service level values were assigned to each greenway class in the order of natural numbers. As a result, the relative service level values of Levels 1, 2, and 3 greenways were set to 3, 2, and 1, respectively.

A buffer attribute table can be established through the presented calculation and assignment process (Table 1). The buffer zones of the greenway service were created on the basis of Table 1.

Table 1. Buffer attribute table.

\begin{tabular}{cccc}
\hline Greenway Class & Time of Arrival & Service Radius & Relative Service Level Value \\
\hline Level 3 greenway & $30-45 \mathrm{~min}$ & $2700 \mathrm{~m}$ & 1 \\
Level 2 greenway & $15-20 \mathrm{~min}$ & $1200 \mathrm{~m}$ & 2 \\
Level 1 greenway & $5-10 \mathrm{~min}$ & $600 \mathrm{~m}$ & 3 \\
\hline
\end{tabular}

The three-level greenway service buffer zones were overlaid by the UNION function of GIS spatial analysis. The relative service level value within the overlapping area was then added to calculate the actual service level value of the greenway buffer. In the relative service level value in Table 1 , the actual service level value is an integer between 1 and 6 . The superposition results were then divided into three categories. The service levels were set to III, II, and I when the actual service level values ranged between 1 and 2, 3 and 4, and 5 and 6, respectively. Thus, the distribution map of the greenway service level was obtained (Figure 5). 


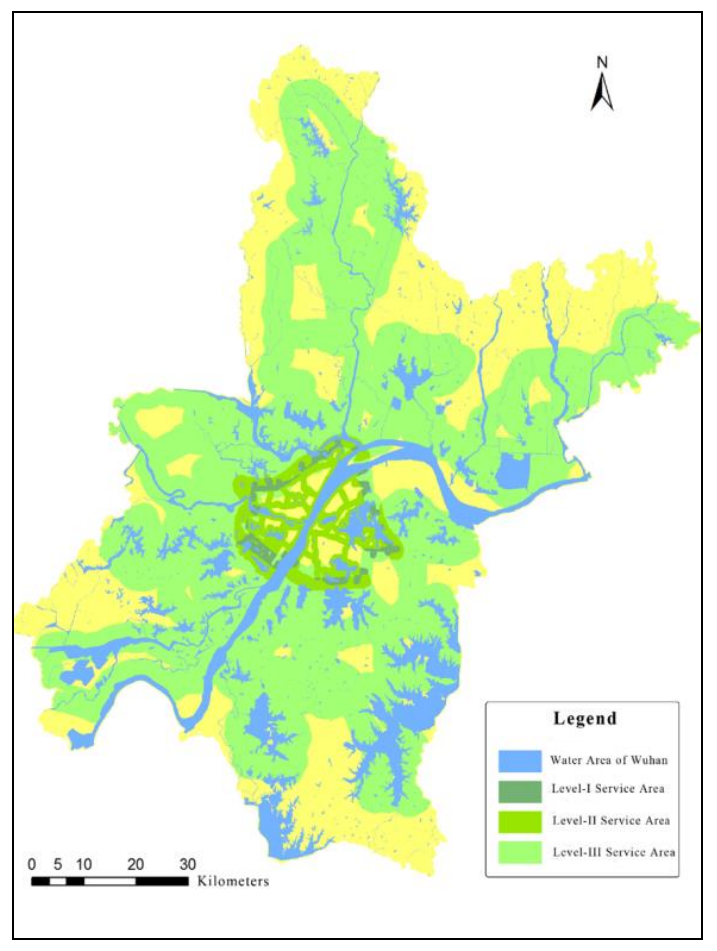

Figure 5. Spatial distribution map of the service levels of the greenway network in Wuhan.

After obtaining the greenway service level data shown in Figure 5, the population density distribution map was generated using the sixth census, Data of Wuhan. The population density distribution of Wuhan is shown in Figure 6.

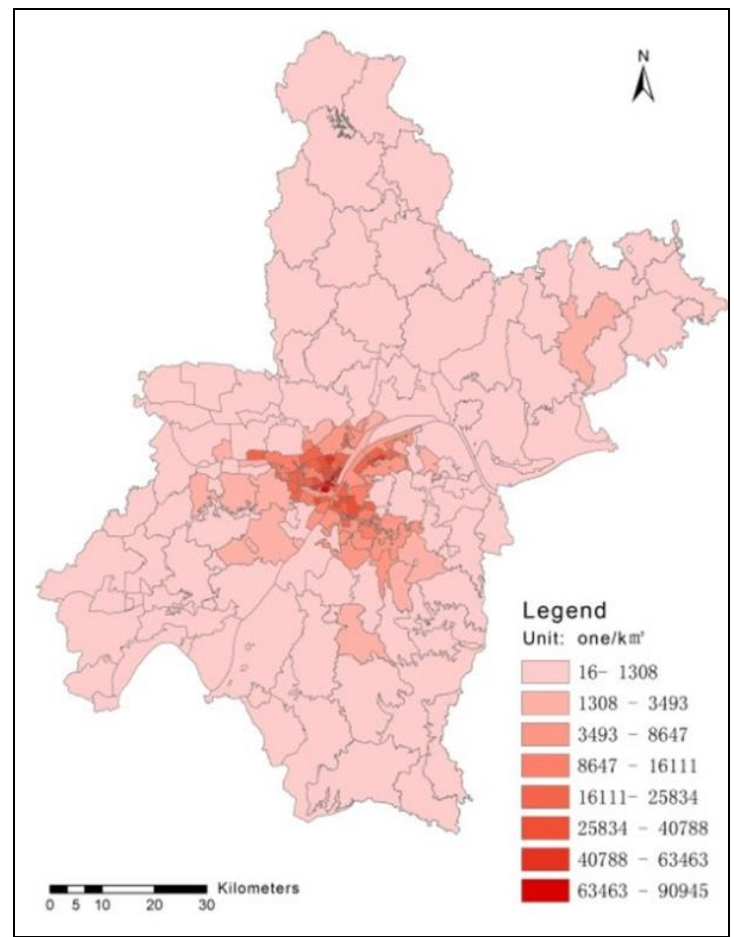

Figure 6. Spatial distribution map of the population density of Wuhan. 
The greenway service level data and population density data were intersected to obtain the demographic information of different service levels by using the intersection function of ArcGIS overlay analysis module. The greenway service level data and the Wuhan residential quarter data were superimposed to obtain the living area statistics in different service levels. The number of urban populations and the area of residential quarters were determined by following the abovementioned detailed steps, and our results indicated that urban greenways can serve the entire city area.

\subsection{Minimum Distance-Based Accessibility Analysis}

Service convenience is another important aspect of service capability evaluation, and this aspect reflects the convenience of urban residents to utilize greenway services. Therefore, the service convenience of urban greenways should be analyzed to evaluate their service capacity. The reachability analysis method, based on the minimum proximity distance, is based on the distance from the residential area to the green road, which can effectively show the convenience of the greenway service. This method is used in our study on the basis of the service convenience, to evaluate the service capacity of urban greenways.

The minimum distance method represents the accessibility level by considering the distance from the residential quarters to the nearest greenway. This method is based on the assumption that people tend to exercise or spend their leisure times at greenways located close to their living area. Thus, this method is appropriate and commonly used in accessibility analysis [16].

A rational level division of greenway accessibility should be formed in the implementation stage. In accordance with the Wuhan greenway system planning stated in Section 2, the public can reach the greenways in all regions within 5-45 min. A 10 min interval was set for segmentation of the arrival times, to present the accessibility level of greenway. Hence, the total arrival time interval was divided into five segmentations, which consisted of 5-15, 15-25, 25-35, 35-45, and more than $45 \mathrm{~min}$. Based on these arrival time ranges, the normal walking speed is assumed to be $1 \mathrm{~m} / \mathrm{s}$. The ranges of reach distance were then determined for each corresponding time segment. These ranges are shorter than 900, 900-1500, 1500-2100, 2100-2700, and longer than $2700 \mathrm{~m}$.

The accessibility level division table can be established by using the presented division and estimation processes (Table 2). In Table 2, a short reach distance corresponds to a high accessibility level. Thus, the highest accessibility level is Level I, and the lowest accessibility level is Level V.

In this method, the distance from the residential quarters to the nearest greenway was calculated by using the minimum distance module of ArcGIS. The residential quarters were subsequently divided into different accessibility levels based on the residential quarter data of Wuhan, and the division rules shown in Table 2. Based on the distribution of residents of Wuhan (shown in Figure 6), and requirements of different accessibility level in time and distance (shown in Table 2), we know that the greenway which meets the time and distance requirements of different accessibility levels is mainly located in the main urban area of Wuhan. The obtained distribution thematic map of the greenway accessibility level in main urban area of Wuhan is shown in Figure 7.

Table 2. Accessibility level division table.

\begin{tabular}{ccc}
\hline Accessibility Levels & Time of Arrival & Reach Distance \\
\hline Level I & $5-15 \mathrm{~min}$ & $<900 \mathrm{~m}$ \\
Level II & $15-25 \mathrm{~min}$ & $900-1500 \mathrm{~m}$ \\
Level III & $25-35 \mathrm{~min}$ & $1500-2100 \mathrm{~m}$ \\
Level IV & $35-45 \mathrm{~min}$ & $2100-2700 \mathrm{~m}$ \\
Level V & $>45 \mathrm{~min}$ & $>2700 \mathrm{~m}$ \\
\hline
\end{tabular}

Finally, a statistic of the residential quarter area in different greenway accessibility levels can be performed using the greenway accessibility level data (Figure 7). Then, the greenway accessibility level data and the population density data were intersected to obtain the demographic information 
of different accessibility levels. Through these processes, we determined the area of residential quarters and the number of residential population at different levels of accessibility. Obviously, the higher the accessibility level, the more conveniently the residents can reach a greenway from their residential quarters.

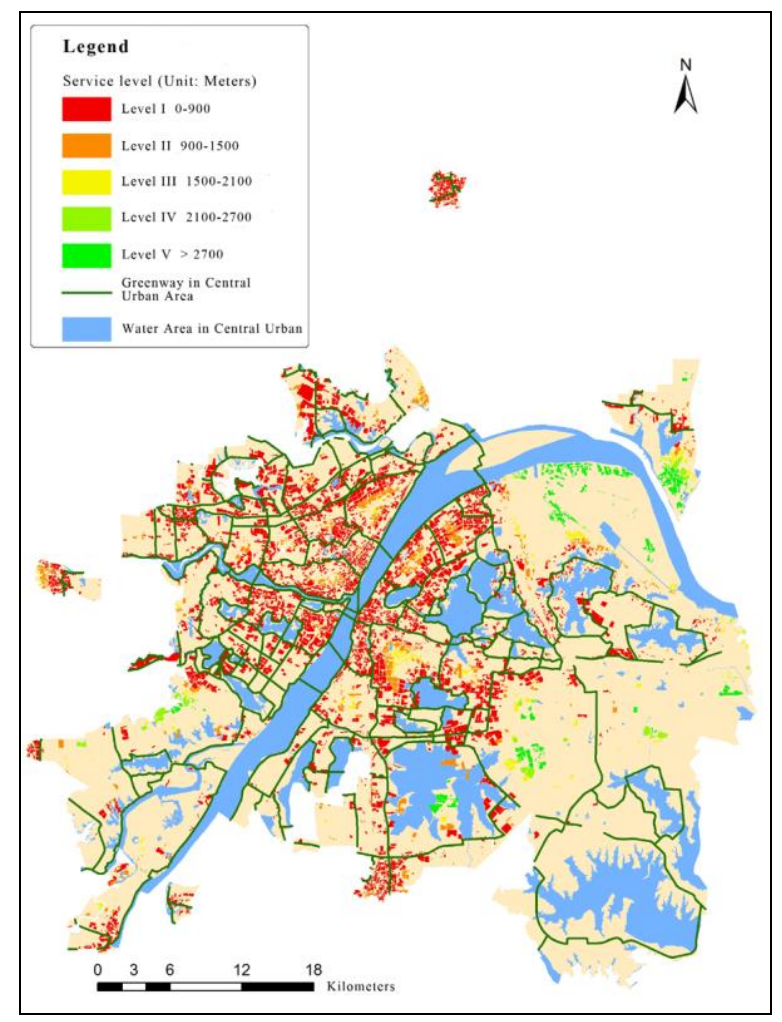

Figure 7. Distribution thematic map of the greenway accessibility level in main urban area of Wuhan.

\subsection{Comprehensive Service Capability Evaluation}

Quality of service is another important aspect of service capacity evaluation, and this parameter indicates the actual effect of a greenway service. Therefore, the quality of services should be assessed to evaluate the service capacity of urban greenways. Integrated service capability analysis method combines various influencing factors that can objectively reflect the actual effect of greenway service. This method is used on the basis of service quality perspectives to evaluate the service capacity of urban greenways.

Relevant factors should be defined to evaluate the comprehensive service capability of an urban greenway network. In our study, the following factors used to evaluate the comprehensive service capability of the greenway network of an urban area are proposed: urban traffic convenience, public and government service facilities surrounding the greenway, urban population density, residential areas, and greenway service level values. The first five factors are called influencing factors. Thus, this study presents a greenway comprehensive service capability model based on regular grids. The calculation formula is given as follows:

$$
A_{i}=\left(2 p_{i}+2 r_{i}+s_{i}+g_{i}+t_{i}\right) \times L_{i}
$$

where ${ }_{i}$ is the comprehensive service capability of the $i$-th grid, $p_{i}$ is the population coefficient of the $i$-th grid, $r_{i}$ is the residential area coefficient of the $i$-th grid, $s_{i}$ is the public service facility coefficient of the $i$-th grid, $g_{i}$ is the government service facility coefficient of the $i$-th grid, $t_{i}$ is the public transport coefficient of the $i$-th grid, and $L_{i}$ is the greenway service level coefficient of the $i$-th grid. 
In reality, the service objects of greenways are mainly the residents of urban areas. Among the five influencing factors, population and residential area coefficients represent the number of potential greenway users, which is the main aspect influencing the quality of green service. Public transport, public service facility, and government service facility coefficients correspond to the completeness of greenway service, and their influence on the quality of greenway service is lower than that of population and residential area coefficients. The weight of the main influencing factor is set to 2 , because the value range of the influencing factor is at $[0,1]$ to show the difference of the degree of influence accurately. The weight of the secondary influencing factor is set to 1 . In Equation (2), other modest weigh settings are equally acceptable, as long as they do not overstate or diminish the degree of influence.

Six key steps should be performed on the basis of the model shown in Equation (2), to derive the comprehensive service capability of urban greenways.

(1) Regular grid division.

In Step 1, the research area was divided into regular grids with the size of $1 \mathrm{~km} \times 1 \mathrm{~km}$, based on the construction goal of "500 Meters to Reach the Greenbelt, 1000 Meters to Reach the Garden."

(2) Value statistics of the five influencing factors in each grid cell.

In Step 2, the values of the five influencing factors include the number of population in a grid cell $\left(G r i d P_{n}\right)$, the area of residential quarters in a grid cell $\left(G r i d R e s_{n}\right)$, the number of public transport points in a grid cell $\left(G r i d C T_{n}\right)$, the number of public service points in a grid cell $\left(G r i d C S_{n}\right)$, and the number of government service points in a grid cell $\left(G r i d C G_{n}\right)$. We suppose that $R$ represents a single grid cell, $P_{n}$ represents the population, $A_{n}$ represents the area of residential quarters, $T_{n}$ represents the position of public transport points, $S_{n}$ represents the position of public service points, and $G_{n}$ represents the position of government service points. The processes of value statistics were as follows: The first process involves overlaying the regular grid layer to the layers related to the population, residential quarter, public transport, public service, and government service. The second involves calculating the values of the five influencing factors in each grid cell using Equation (3).

$$
\left\{\begin{array}{c}
\operatorname{GridP}_{n}=\sum P_{n} \in \mathrm{R} \\
\operatorname{GridRes}_{n}=\sum A_{n} \in R \\
{\operatorname{Grid} C T_{n}}=\sum T_{n} \in R \\
\operatorname{GridCS}_{n}=\sum S_{n} \in R \\
\operatorname{GridC}_{n}=\sum G_{n} \in R
\end{array}\right.
$$

In Equation (3), $X \in R$ means that the spatial objects are within a grid cell.

(3) Grading of the five influencing factors.

In Step 3, the thematic map of the distribution of the five influencing factors (Figure 8) is derived by grading the values of the five influencing factors in each grid cell, which were obtained in Step 2 . A high value of a certain influencing factor likely yields a high grade of the corresponding influencing factor. In Figure 8, the distribution of five different influencing factors indicate that the high-grade areas are concentrated in the urban center. Low-grade areas are widely distributed throughout the suburban areas.

(4) Calculation of the coefficients of the five influencing factors.

In Step 4, weights were assigned to each corresponding grade of each influencing factor in the order of natural numbers (e.g., grade 1 had an assigned weight of 1, grade 2 had an assigned weight of 2) after grading for the five influencing factors. We then normalized the weights to obtain the final coefficients. Equation (4) shows the calculation method of weight normalization.

$$
E_{i} \cong \frac{Q_{i}}{\sum_{i=1}^{n} Q_{i}},
$$

In Equation (4), $E_{i}$ represents the coefficient of grade $i$ of a certain influencing factor, $Q_{i}$ represents the assigned weight of grade $i$ of a certain influencing factor, $i$ represents the grade of a certain influencing factor, and $n$ represents the highest grade of a certain influencing factor. 


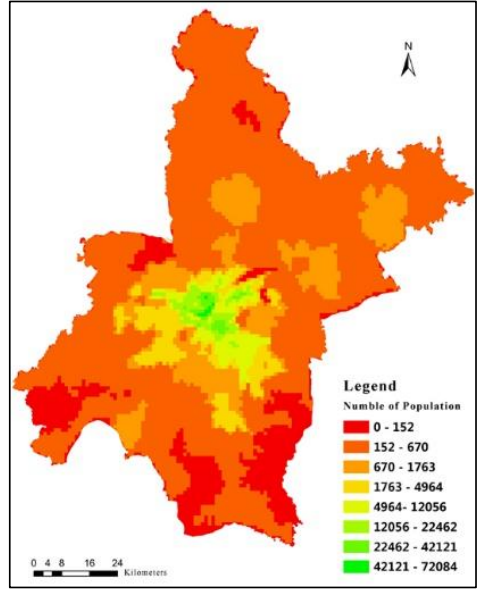

(a)

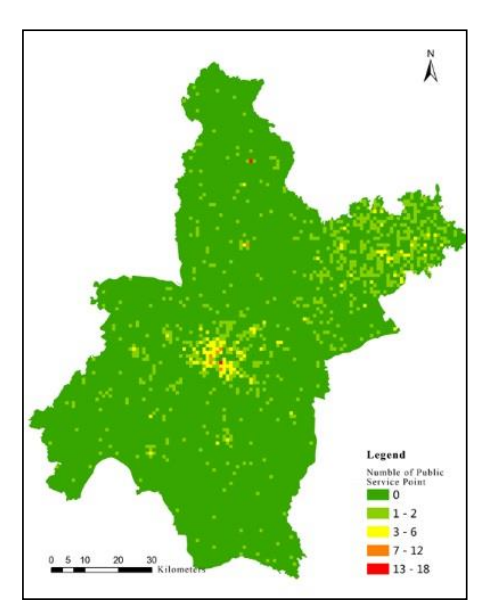

(d)

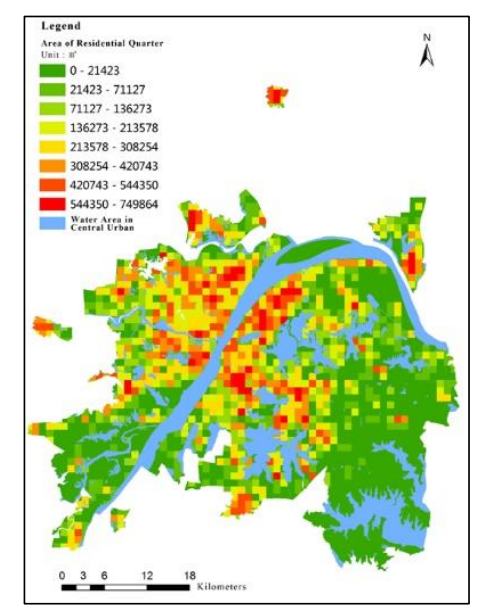

(b)

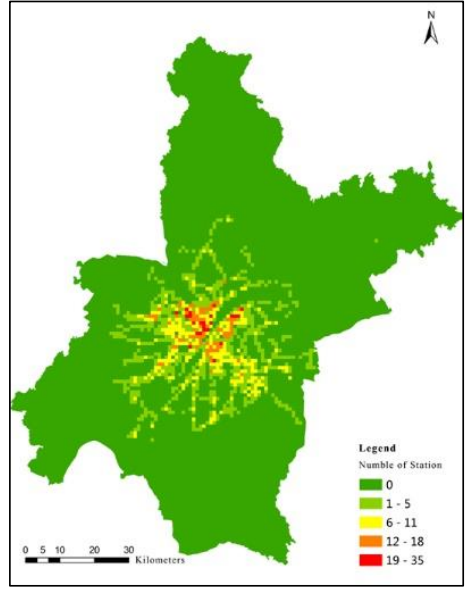

(c)
Figure 8. Thematic map of the distribution of the five influencing factors.

(5) Calculation of the greenway service level coefficient.

In Step 5, the greenway service level data, including the actual service level value, obtained in Section 3.2 were used. First, the regular grid layer and the greenway service level layer (data) were overlaid. Next, the average of the actual service level value in each grid cell was calculated, that is, the average of a certain grid cell was the greenway service level coefficient of that grid cell. $R$ represents a single grid cell, $L_{i}$ reflects the greenway service level coefficient of a certain grid cell, $l_{i}$ corresponds to one of the actual service level value in a certain grid cell, and $C_{i}$ refers to the number of the actual service level value in a certain grid cell. The greenway service level coefficient is calculated using Equation (5).

$$
L_{i}=\frac{\sum l_{i} \in R}{C_{i}}
$$

In Equation (5), $X \in R$ means that the spatial objects are within a grid cell.

(6) Calculation of the comprehensive service capability value of urban greenways.

In Step 6, the coefficients of the five influencing factors obtained in Step 4, and the greenway service level coefficient obtained in Step 5, were substituted into Equation (2) to derive the comprehensive service capability value of urban greenways in each grid cell. A high value of $A_{i}$ represents high service quality. 


\section{Results and Analysis}

\subsection{Evaluation of Service Level and Accessibility Level of Urban Greenways in Wuhan}

In service level evaluation, the buffer-based service level analysis method was initially applied to the Wuhan greenway network, and the number of service population and the area of service residential quarters in each service level were then obtained. In accordance with these data, the service population [17] and service residential quarter area ratios [Equations (6) and (7)] were computed as the basis of the quantitative evaluation of greenway service level in the study area by using the following equations.

$$
\begin{aligned}
& R_{p}=S_{p} / P \times 100 \% \\
& R_{a}=S_{a} / A \times 100 \%
\end{aligned}
$$

In Equations (6) and (7), $R_{p}$ represents the service population ratio, $S_{p}$ refers to the service population, $P$ denotes the total population of the study area, $R_{a}$ corresponds to the service residential quarter area ratio, $S_{a}$ indicates the service residential quarter area, and $A$ shows the total residential quarter area.

$S_{p}$ and $S_{a}$ were obtained using the buffer-based service level analysis method stated in Section 3.2. $P$ was obtained from the population density data and $A$ was obtained from the residential quarter data. The statistical results are shown in Table 3.

Table 3. Statistical table of greenway service level.

\begin{tabular}{ccccc}
\hline Service Level & Level I & Level II & Level III & Total \\
\hline Service population & 455,123 & $3,441,018$ & $3,285,129$ & $7,181,270$ \\
Service population ratio (\%) & 4.65 & 35.16 & 33.57 & 73.38 \\
Service residential quarter area $\left(\mathrm{km}^{2}\right)$ & 13.68 & 73.37 & 105.97 & 193.02 \\
Service residential quarter area ratio $(\%)$ & 5.65 & 30.33 & 43.81 & 79.79 \\
\hline
\end{tabular}

Table 3 shows that the Wuhan greenway network has excellent service capability and wide scope of service. The city has a service population ratio of $73.38 \%$ and a service residential quarter area ratio of $79.79 \%$. These results indicate that the vast majority of the residents and residential quarters of Wuhan can access the greenway service. However, the population and the area of the residential quarters with high-level greenway service remain insufficient. Level I and Level II areas with high-quality service can only serve $4.65 \%$ and $35.16 \%$ of the population, and cover only $5.65 \%$ and $30.33 \%$ of the residential quarter areas, respectively. Therefore, the urban greenway system of Wuhan and the greenways in the main urban areas should be further improved.

In the aspect of accessibility level evaluation, we first applied the minimum distance-based accessibility analysis method to evaluate the Wuhan greenway network. In doing so, we obtained the approximate residential population and the residential quarter areas under different accessibility levels. In accordance with these results, the residential population ratio shown in Equation (8), and the residential quarter area ratio shown in Equation (9), were further computed as the basis of quantitative evaluation of greenway accessibility level in the residential area using the equations below.

$$
\begin{aligned}
& r_{p}=s_{p} / p \times 100 \%, \\
& r_{a}=s_{a} / A \times 100 \%,
\end{aligned}
$$

In Equation (8), $r_{p}$ represents the residential population ratio under a certain accessibility level, $s_{p}$ is the approximate residential population under an accessibility level, and $p$ is the approximate total residential population. In Equation (9), $r_{a}$ represents the residential quarter area ratio under a certain accessibility level, $s_{a}$ is the residential quarter area under an accessibility level, and $A$ is the total residential quarter area. 
$s_{p}$ and $s_{a}$ were obtained using the minimum distance-based accessibility analysis method stated in Section 3.3. $p$ was obtained by intersecting the greenway accessibility level data with the population density data and $A$ was obtained from the residential quarter data. The statistical results are shown in Table 4 .

Table 4. Statistical table of greenway accessibility level.

\begin{tabular}{cccccc}
\hline Accessibility Level & Level I & Level II & Level III & Level IV & Level V \\
\hline Approximate residential population & $1,594,214$ & 234,994 & 39,823 & 10,206 & 14,694 \\
Residential population ratio $(\%)$ & 84.17 & 12.41 & 2.10 & 0.54 & 0.78 \\
Residential quarter area $\left(\mathrm{km}^{2}\right)$ & 184.18 & 26.84 & 9.96 & 6.58 & 14.32 \\
Residential quarter area ratio $(\%)$ & 76.15 & 11.10 & 4.12 & 2.72 & 5.92 \\
\hline
\end{tabular}

Table 4 shows that $76.15 \%$ of the residential quarter area is in the $0-900 \mathrm{~m}$ range of Level I, covering $84.17 \%$ of the residential population. About $11.1 \%$ of the residential area is in the $900-1500 \mathrm{~m}$ range of Level II, covering $12.41 \%$ of the residential population. Overall, the residential quarters comprise $87.25 \%$ of the total residential quarter area in the $1500 \mathrm{~m}$ range from the residential quarters to the nearest greenways. Moreover, the total number of the residential population accounted for $96.58 \%$. The results illustrate that most of the residential quarters in Wuhan have the highest level of greenway accessibility. In other words, the vast majority of community residents can easily reach the greenways surrounding them. Hence, the greenways of Wuhan have a high convenience level.

\subsection{Evaluation of the Comprehensive Service Capability of Urban Greenways in Wuhan}

Combined with the data on population density, residential quarters, public transport points, public service points, government service points, and greenway service level, the six coefficients of each grid cell were obtained according to the key steps described in Section 3.4. The coefficients of each grid were then considered in the greenway comprehensive service capability model shown in Equation (2). Thus, the greenway comprehensive service capability value of each grid was calculated. The calculated results were subsequently divided into four grades, and the distribution map of the comprehensive service capability of the greenways in Wuhan was obtained. A thematic map of the main urban area of Wuhan is presented in Figure 9.

Figure 9 shows that the comprehensive service capability changes from a strong level to a weak level, as green turns red. Figure 9 also indicates that the strong comprehensive service capability area of the Wuhan urban greenway system is mainly concentrated in scenic areas, such as the Yellow Crane Tower, Donghu, Moshan, and Zhongshan Park, and in the confluence area of the Yangtze and Han rivers. The comprehensive service capability of the greenway is relatively low in far urban areas. Finally, the land area of each grade in the central urban area is determined, and the statistical results are shown in Table 5.

In Table 5, Grade III comprehensive service capability in the central urban area comprises $69.12 \%$ of the total area. Thus, the coverage area is wide. In Figure 9, these areas are mainly distributed around the urban center. The comprehensive service capability grades of the urban greenway network in the urban center are mainly Grades I and II. The areas with Grades I and II account for $7.35 \%$ and $13.84 \%$, respectively. Overall, the space distribution of the comprehensive service capability of the urban greenway system in Wuhan shows that features are strong in the center, and weak in the suburbs. Moreover, Grade I and II areas, with strong comprehensive service capabilities, are mainly distributed along the core scenic areas, and thus satisfy the functional requirements of greenways, such as connecting scenic spots, improving urban ecological environments, providing sightseeing and leisure places for the public, and protecting relevant historical and cultural resources. 


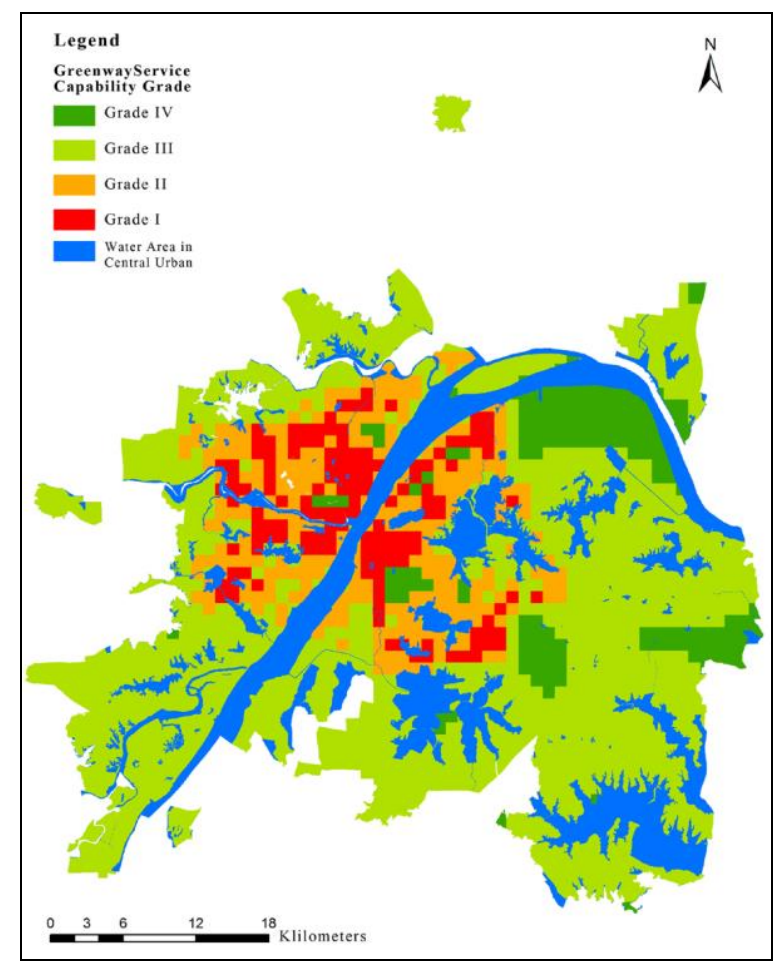

Figure 9. Distribution map of the different grades of the comprehensive service capability of the greenway network in main urban area of Wuhan.

Table 5. Statistical table of the different grade areas of the greenway network's comprehensive service capability of main urban area of Wuhan.

\begin{tabular}{ccc}
\hline Comprehensive Service Capability Grade & Land Area $\left.\mathbf{( k m}^{\mathbf{2}}\right)$ & Land Area Ratio $\mathbf{( \% )}$ \\
\hline Grade I & 140.78 & 7.35 \\
Grade II & 265 & 13.84 \\
Grade III & 1323.27 & 69.12 \\
Grade IV & 185.46 & 9.69 \\
\hline
\end{tabular}

\section{Conclusions}

Planning and constructing an appropriate greenway network are very important components of modern urban development. Greenway network construction constitutes the main building project of many cities in the world. However, few studies on service capability evaluation of urban greenways have been reported.

In this paper, the Wuhan urban greenway system was considered as our case study. With GIS technology and geographical spatial data, this study proposed an approach to evaluate the overall service capability of urban greenways in three different aspects. The proposed spatial analysis-based approach can be a valuable option to assess the urban greenway service capability, and simultaneously quantify and spatialize the abstract service capability.

In the first aspect, the urban greenway system of Wuhan yields a service population ratio of $73.38 \%$, and a service residential area ratio of $79.79 \%$. This result indicates that the majority of residents and residential quarters in Wuhan can access the greenway service. However, Level I and Level II areas with high-quality service, can respectively serve $4.65 \%$ and $35.16 \%$ of the population and cover $5.65 \%$ and $30.33 \%$ of the residential quarter areas. These findings suggest that the high-level greenway service in Wuhan remains insufficient. To overcome this shortcoming, we propose in Figure 5, a possible 
approach that can assist greenway planners in locating service blind zones in the main urban area, and thus ensure that greenway supplement strategies can be introduced.

In the second aspect, residential quarters account for $87.25 \%$ of the total residential quarter area within the $1500 \mathrm{~m}$ range from the residential quarters to the nearest greenways. This result implies that urban greenways in Wuhan provide a high convenience level. However, the residential quarters with the low accessibility of Level IV or Level V are easily located (Figure 7). This result can help decision-makers focus on this part of residential quarters and implement accurate decisions to improve the convenience of urban greenways.

In the third aspect, Grade III and IV areas, with relatively weak comprehensive service capabilities, account for $78.81 \%$. However, Grade I and II areas, with strong comprehensive service capabilities, correspond to $21.19 \%$. This result reveals that the comprehensive service capability of Wuhan greenways is not balanced between the main urban area and the far urban area. To reduce this regional imbalance, we illustrate in Figure 9, a potential method that can help urban planners and decision-makers determine the limitations of greenway services, and create optimized urban greenway designs.

Acknowledgments: The authors greatly appreciate the Wuhan Geomatic Institute for providing the detailed experimental data used in the study. We would also like to thank the reviewers for their helpful comments and suggestions, which improved the presentation of this paper. This work was supported by the Key Project of NSFC (No. 41331175).

Author Contributions: Penglin Zhang conceived the study; all co-authors formulated and designed the methodology. Junwen Bai performed the main experiments; Penglin Zhang and Junwen Bai wrote the paper; Zheng Li and Juan Du performed part experiments and paper revision. All authors have read and approved the final manuscript.

Conflicts of Interest: The authors declare no conflict of interest.

\section{References}

1. Lu, F.; Yin, H.; Kong, F. The Using Characteristics and Satisfaction of Urban Greenway-A Case Study of the Purple Mountain Greenway in Nanjing. Chin. Landsc. Archit. 2015, 31, 50-54.

2. President's Commission on Americans Outdoors. Americans Outdoors: The Legacy, the Challenge, with Case Studies; Island Press: Washington, DC, USA, 1987.

3. Little, C. Greenway for American; Johns Hopkins University Press: Baltimore, MD, USA, 1990; pp. 7-20.

4. Zhou, N.; Yu, K.; Huang, Z. Perspectives on Greenway Development. Acta Ecol. Sin. 2006, 26, 3108-3116.

5. Zeng, G. The Service Level Evaluation of Urban Parks-A Case Study in Wuhan; Wuhan University: Wuhan, China, 2011.

6. Tan, S.; Zhao, W. Progress and Prospect of Greenway Planning and Research. Chin. Landsc. Archit. 2007, 23, 85-89.

7. Hu, J.; Dai, F. Progress of Greenway Research in China. Chin. Landsc. Archit. 2010, 26, 88-93.

8. Yahner, T.G.; Korostoff, N.; Johnson, T.P.; Battagila, A.M.; Jones, D.R. Cultural landscape and landscape ecology in contemporary greenway planning, design and management: A case study. Landsc. Urban Plan. 1995, 33, 295-316. [CrossRef]

9. Miller, W.; Collins, M.G.; Steiner, F.R.; Cook, E. An approach for greenway suitability analysis. Landsc. Urban Plan. 1998, 42, 91-105. [CrossRef]

10. Lindsey, G. Use of urban greenways: Insights from Indianapolis. Landsc. Urban Plan. 1999, 45, 145-157. [CrossRef]

11. Conine, A.; Xiang, W.N.; Young, J.; Whiyley, D. Planning for multi-purpose greenway in Concord, North Carolina. Landsc. Urban Plan. 2004, 68, 271-287. [CrossRef]

12. $\mathrm{Wu}, \mathrm{J}$. Post Occupancy Evaluation (POE) on the Zengcheng Greenway System in Guangdong Province. Chin. Landsc. Archit. 2011, 17, 39-43.

13. Lin, J.; Gan, Q.L.; Wei, S.; Zhang, T.J. Guang Zhou Greenway Function Perception of IPA Assessment and Analysis. Yunnan Geogr. Environ. Res. 2012, 3, 14-18. 
14. Zhang, X.L. Post Occupancy Evaluation on the Star Lake Greenway in Zhao Qing. Trop. Geogr. 2012, 4, 429-436.

15. Li, B. Study of Greenway Planning and Construction Outside the 3rd Ring Rd of Chengdu Based on Users' Satisfaction; Southwest Jiaotong University: Chengdu, China, 2013.

16. Wang, Y.Q. Accessibility and Service Assessment on Urban Park Green Space of Tianjin City. J. Anhui Agric. Sci. 2013, 41, 1616-1618.

17. Yang, W.K. Research on Service Level of Green Spaces Based on GIS and RS of Hang Zhou; Zhejiang University: Hang Zhou, China, 2014.

(C) 2017 by the authors. Licensee MDPI, Basel, Switzerland. This article is an open access article distributed under the terms and conditions of the Creative Commons Attribution (CC BY) license (http:// creativecommons.org/licenses/by/4.0/). 